\title{
NeuroRegulation
}

\section{A Meta-Analysis of the Effect of Neurofeedback on Depression}

\author{
Demir Barlas \\ Integrative Wellness Upstate NY, Ithaca, New York, USA
}

\begin{abstract}
This meta-analytical study examined the effect of neurofeedback (NF) on decreasing depression. The main finding of the meta-analysis was that NF resulted in a large $(g=-0.91)$ decrease in depression. This finding can be explained through the various roles of brainwave oscillations in terms of both the formation and persistence of depression and the development of oscillatory patterns less compatible with depressive states. One plausible mechanism for NF's depression-reduction effect is that of the approach-withdrawal model as related to not only the asymmetrical activation of the frontal regions but also the hypoactivity of the amygdala. Future research might uncover other possible explanations for NF's observed efficacy as a means of reducing depression. The findings of the study provide some support for the utilization of NF as either a complement to the pharmaceutical treatment of depression or, given its effect size, a standalone therapy. However, because NF research base is immature in comparison to the research base on pharmaceutical antidepressants, additional analysis remains necessary.
\end{abstract}

Keywords: neurofeedback; depression; biofeedback; arousal-withdrawal model; frontal alpha asymmetry; amygdala

Citation: Barlas, D. (2021). A meta-analysis of the effect of neurofeedback on depression. NeuroRegulation, 8(2), 104-111. https://doi.org/10.15540/nr.8.2.104

*Address correspondence to: Demir Barlas, Integrative Wellness Upstate NY, South Hill Business Campus, 950 Danby Rd, Ste 202-G, Ithaca, NY 14850. Email: demirbarlas@gmail.com

Copyright: (c) 2021. Barlas. This is an Open Access article distributed under the terms of the Creative Commons Attribution License (CC-BY).
Edited by:

Rex L. Cannon, PhD, SPESA Research Institute, Knoxville, Tennessee, USA

\section{Reviewed by:}

Rex L. Cannon, PhD, SPESA Research Institute, Knoxville, Tennessee, USA

Randall Lyle, PhD, Mount Mercy University, Cedar Rapids, lowa, USA

\section{Introduction}

In 2017, 264 million people globally had some form of depressive disorder (James et al., 2018). The prevalence of major depressive disorder (MDD) has been between 4 and $4.7 \%$ from 1990 onwards (Baxter et al., 2014). Depression is a costly disease in terms of its impacts on (a) medical expenses, particularly in the case of treatment-resistant depression (TRD; Shin et al., 2020); (b) workplace productivity (Bubonya et al., 2017); and (c) decreased quantity and quality of life (Jia et al., 2015). For these and other reasons, there is an abiding public health interest in decreasing the prevalence and intensity of depression.

Globally, pharmaceutical treatments of depression are common (Shin et al., 2020), but there is also growing evidence for the potential efficacy of nonpharmaceutical treatments such as psychotherapy, meditation/mindfulness, and biofeedback (BF), as in the form of neurofeedback (NF). Nonpharmaceutical approaches are particularly promising in cases of TRD, which are both more costly (Shin et al., 2020) and, by definition, more difficult to resolve through accepted treatments. Increasing interest in nonpharmaceutical approaches to treating depression has resulted in numerous studies on the possible effectiveness of NF as a depression-reducing modality.

As the body of studies on NF grows, a meta-analysis is capable of providing a more accurate estimate of the effect of NF on depression while also isolating themes related to the effectiveness of NF that require additional research attention. The two purposes of this meta-analysis are to (a) calculate a Hedges' $g$ measure of effect size for the effect of NF 
on depression symptom reduction and (b) discuss possible reasons for the observed effectiveness of NF as a nonpharmaceutical approach to the reduction of depression. The results are of interest as not only a contribution to the meta-analytical literature on NF but also as a means of identifying and discussing the theoretical reasons for NF's possible antidepressant properties.

\section{Methods}

Study data were collected with the objective of deriving a point estimate and accompanying 95\% confidence interval $(C l)$ for the effect of $\mathrm{NF}$ on depression, as measured by Hedges' $g$, a commonly preferred measure of effect size (Peng \& Chen,
2014). Figure 1 below is the PRISMA flow diagram for the meta-analysis, with the primary database source for articles being PUBMED. The primary reason for study exclusion was failure to report both depression means and standard deviations for before- and after-NF conditions.

\section{Results}

Table 1 below contains a summary of the included studies $(k=11)$. Some studies contained more than one NF protocol, allowing more than one result to be extracted. The Beck Depression Inventory (BDI-II; Beck et al., 1996) was used in all but one of the included studies.

Figure 1. PRISMA Flow Diagram for Meta-analysis.

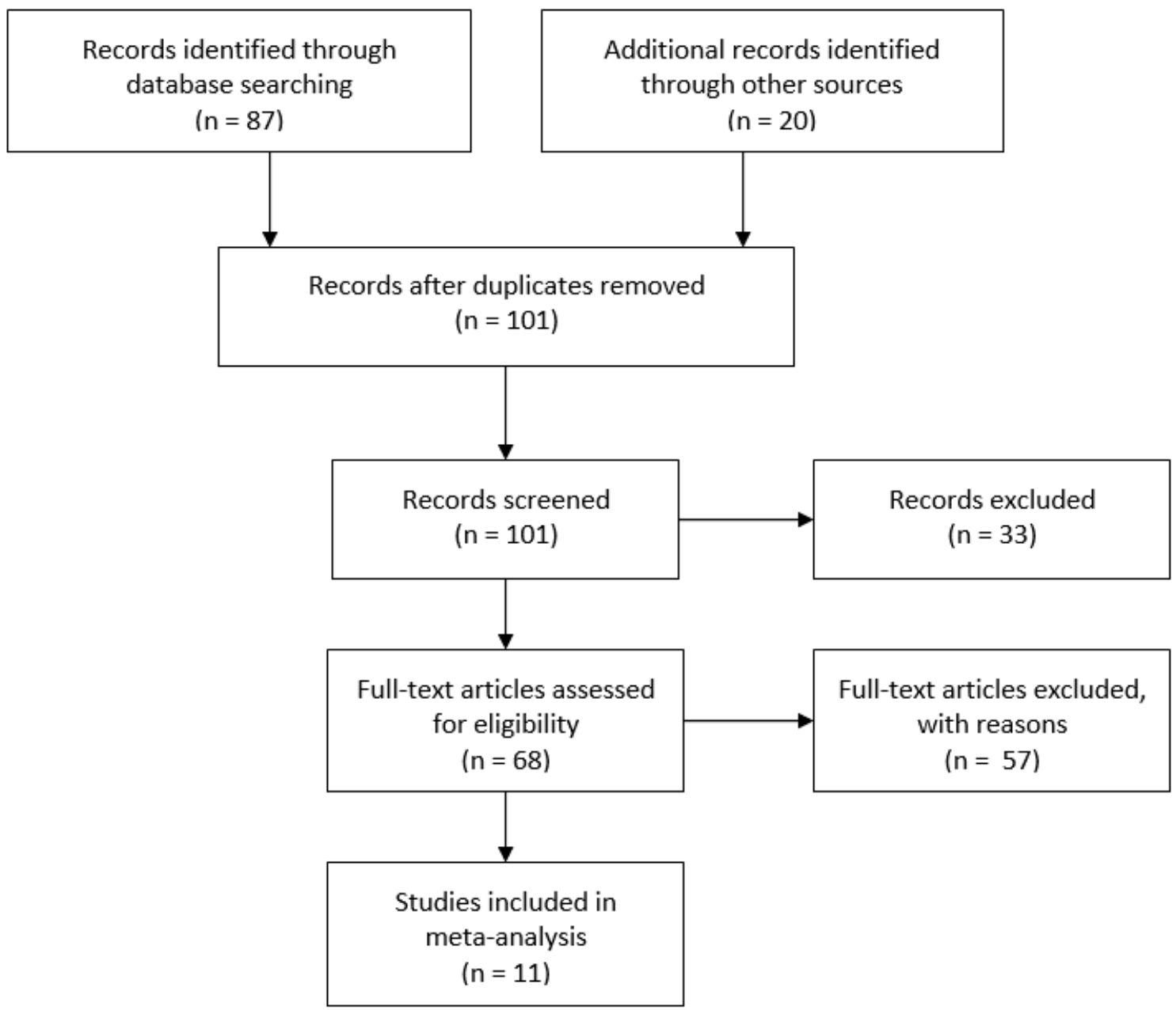


Table 1

Summary of Studies in Meta-Analysis

\begin{tabular}{|c|c|c|c|c|c|}
\hline Citation & $\begin{array}{l}\text { Depression } \\
\text { (D) Measure }\end{array}$ & $\begin{array}{l}\text { D Before NF: } \\
\qquad M(S D)\end{array}$ & $\begin{array}{l}\text { D After NF: } \\
M(S D)\end{array}$ & $\begin{array}{l}\text { Sample Size (Control) } \\
\text { and Description }\end{array}$ & NF Protocol \\
\hline Yu et al. (2020) & BDI-II & $24.90(8.25)$ & $16.80(9.24)$ & $\begin{array}{l}14 \text { (Chinese } \\
\text { university students) }\end{array}$ & $\begin{array}{l}\text { 10-11 Hz uptraining } \\
\text { Fp1 \& Fp2 }\end{array}$ \\
\hline Young et al. (2017) & BDI-II & $27.20(10.70)$ & $16.10(9.70)$ & 18 (American adults) & rftMRI-NF \\
\hline Takamura et al. (2020) & BDI-II & $28.70(8.57)$ & $17.20(6.12)$ & 6 (Japanese adults) & rftMRI-NF \\
\hline $\begin{array}{l}\text { Wang et al. (2019, } \\
\text { second protocol) }\end{array}$ & BDI-II & $29.17(11.47)$ & $17.83(11.20)$ & 23 (Chinese adults) & $\begin{array}{l}\text { High beta } \\
\text { downtraining }\end{array}$ \\
\hline Zotev et al. (2020) & $\begin{array}{c}\text { POMS } \\
\text { Depression }\end{array}$ & $15.40(14.00)$ & $7.75(10.10)$ & 16 (American adults) & rtfMRI-NF \\
\hline Cheon et al. (2016) & BDI-II & $25.25(7.91)$ & $14.63(10.98)$ & $\begin{array}{l}20 \text { (South Korean } \\
\text { adults) }\end{array}$ & $\begin{array}{l}\text { ATR + beta } \\
\text { downtraining }\end{array}$ \\
\hline Harris et al. (2021) & BDI-II & $10.55(9.66)$ & $5.64(6.86)$ & 11 (American adults) & Unspecified \\
\hline Hou et al. (2021) & BDI-II & $20.23(10.47)$ & $12.08(7.33)$ & 13 (Chinese adults) & $\begin{array}{l}\text { Alpha asymmetry, } \\
\text { left parietal lobe }\end{array}$ \\
\hline $\begin{array}{l}\text { Hou et al. (2021, } \\
\text { second protocol) }\end{array}$ & BDI-II & $17.69(7.24)$ & $10.31(5.98)$ & 13 (Chinese adults) & $\begin{array}{l}\text { Alpha asymmetry, } \\
\text { right parietal lobe }\end{array}$ \\
\hline Mennella et al. (2017) & BDI-II & $9.75(12.38)$ & $6.00(7.90)$ & 16 (Italian adults) & Alpha asymmetry \\
\hline Kim \& Kim (2015) & BDI-II & $27.43(7.66)$ & $20.21(7.23)$ & $\begin{array}{c}14 \text { (South Korean } \\
\text { adults) }\end{array}$ & Unknown \\
\hline
\end{tabular}

In the random effects model, the effect of NF on depression was observed to be $g=-0.91(-1.11$, -0.70 ), and this effect was significant at $p<.01$. This $g$ value constitutes an effect that Cohen (2013) described as large and supports the claim that neurofeedback is an effective means of decreasing the symptoms of depression.

\section{Discussion}

One means of approaching the discussion is to explore how and why the following NF protocols might be effective in terms of reducing depression. In addition, particular interest should be paid to the potential role of any specific regions of the brain to which these protocols are directed. The overarching objective of this discussion is to explain the context for the meta-analytical findings and their identification of a large effect of NF on depression reduction. However, such a discussion should also be framed in the context of the standard biomedical account of depression and its treatment, primarily through pharmaceutical means
Pharmaceuticals, Depression, and the New Physics of the Brain

An appropriate means of framing any discussion of the role of pharmaceuticals in depression reduction is to summarize the major subclasses of drugs that serve as antidepressants prior to discussing the possible mechanisms for the effectiveness of such drugs. Lenart and Fekete (2021, p. 286) provided a classificatory scheme that distinguishes between (a) typical antidepressants (comprising monoamine oxidase inhibitors; tricyclic antidepressants; selective serotonin reuptake inhibitors, or SSRIs; and serotonin-norepinephrine reuptake inhibitors, or SNRIs); and (b) atypically antidepressants (including tetracyclic antidepressants; norepinephrinedopamine reuptake inhibitors, or NDRIs; norepinephrine reuptake inhibitors, or NRIs; and serotonin antagonists). Lenart and Fekete, who were particularly interested in angiotensin receptor blockers (ARB) as antidepressants, noted that this class of pharmaceutical has a suite of antiinflammatory, antiapoptopic, and antioxidative properties that are cumulatively neuroprotective. 
Figure 2. Forest Plot, Random Effects Model, Pooled Hedges g for Meta-analysis.

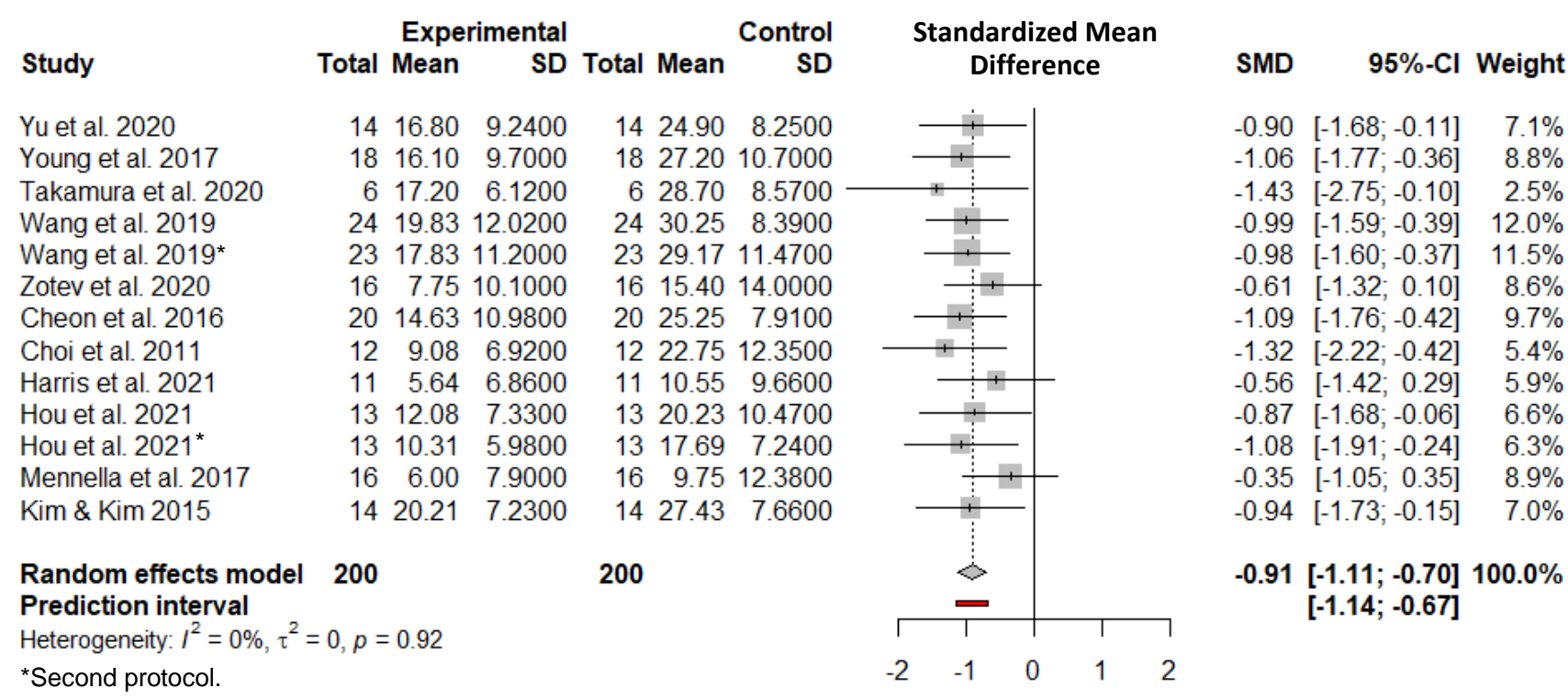

However, summarizing the state of knowledge in 2021, Lenart and Fekete concluded both that there is no integrated and comprehensive account of depression's underlying pathological mechanisms and that "medications are only partially effective" (Lenart \& Fekete, 2021, p. 288).

Notable in Lenart and Fekete's (2021) summary is the identification of many classes of pharmaceuticals, some with highly distinct mechanisms of action. The rationale for the existence of several possible pharmaceutical strategies for treating depression is the complexity of depression itself. To begin with, depression can involve different structures, functions, and pathologies within the brain-for instance, as Kim (2015, p. ix) noted, hypoactivity in the hypothalamicpituitary-adrenal (HPA) axis is correlated more with atypical depression, whereas hyperactivity of the HPA is correlated more with the melancholic subtype of depression. More generally, Kim called attention to the complementary validity of cognitive, biomolecular, and quantum-physical perspectives (see Schwartz et al., 2005) in understanding depression. These perspectives are, as Kim noted, entangled with various complexities in geneenvironment interactions that are specific to individuals who are depressed or at risk for depression.

As both Kim (2015) and Lenart and Fekete (2021) noted, there is consensus on the complexity of depression. Schwartz et al.'s (2005) discussion of quantum physics in relation to neuroscience is relevant to the complexity of any mental disease state. Schwartz et al. noted that the brain cannot be modeled in alignment with the assumptions made by classical physics; quantum physics introduces elements of uncertainty, complexity, and agent choice that prevent, in principle, the application of simple brain-state modeling and the nomological reduction of certain pathologies to certain states of the brain.

Schwartz et al.'s (2005) invocation of the relevance of quantum physics to neuroscience is intended to clarify, not obscure, the neuroscientific research paradigm as it applies to mental illnesses such as depression. Schwartz et al. noted that the function of the brain along quantum-physical as well as classical physical principles means that there are likely to be parallel structures and functions in place of single, reductive pathophysiological pathways. In other words, as Kim (2015) also noted, there will likely never be a single account of depression; it would be more apt to conceptualize depressions in place of depression, with each depression due to various and possibly unique combinations of genetic predisposition, environmental characteristics, agent choice, cognitive strategies, biomolecular function, and structural properties of different parts of the brain.

The pharmaceutical view of treating depression has gone through three phases. In the first phase, the accompanying biomedical paradigm was reductive, with the presumption being that depression is a brain state, or an array of brain states, arising from 
properties of the brain that can be pharmaceutically acted on and cured (Kelly, 2020). In the second phase, TRD and the accumulation of data gave rise to a more complex view of pharmaceutical treatment, one in which a purely reductionistic and mechanistic view of psychopharmacology was challenged (Young \& Moulton, 2020). In the third phase, there is a general acknowledgement (Lenart \& Fekete, 2021) that (a) depression is complex, (b) pharmaceutical approaches are at least partially effective, and (c) there is no unified explanatory account of the effectiveness or ineffectiveness of different pharmacological approaches.

The fundamental existence of uncertainty and parallelism (Schwartz et al., 2005) in neuroscience as a result of quantum-physical challenges to the classical model of physics is not an argument for abandoning the search for physical laws, models, and methods that apply to the brain. However, one result of the paradigm shift in physics (and in neuroscience by way of physics) should be, as Schwartz et al. implied, an epistemic and methodological humbleness in the study of the brain. In a many-paths account of depression such as that of Kim (2015), there is ample room for different ways of studying and treating depression-including, but not limited to, various discrete and overlapping cognitive, molecular, computational, therapeutic, and pharmacological approaches and stances, leading to a multifactorial treatment approach (Sathappan et al., 2019). In such an approach, the guiding research question is not whether nonpharmaceutical approaches to depression work per se; rather, the question is how effective such approaches can be measured to be in terms (such as the $g$ or $d$ measures of effect size) comparable to pharmaceutical approaches.

\section{NF Protocols and Depression}

One of the most widely attested NF protocols for the reduction of depression involves frontal alpha asymmetry reduction. There is also evidence for the effectiveness of real-time fMRI-based NF. Finally, less common NF protocols for depression reduction have also been proposed. The evidence and rationale for several NF protocols are discussed below.

Frontal alpha asymmetry is a state in which there is more activity in the left frontal cortex vis-à-vis the right frontal cortex (Harmon-Jones, 2003). Relatively higher left frontal cortical activity is, in turn, associated with positive affect, whereas relatively higher right frontal cortical activity is associated with negative affect (Tomarken et al., 1992). Frontal alpha asymmetry thus either causes, or is correlated with, the predominance of negative over positive affect. Harmon-Jones in particular is associated with a refinement of this hypothesis, suggesting (Harmon-Jones, 2003; Harmon-Jones et al., 2010) that frontal activation asymmetry should not be understood in terms of affect. This version of the asymmetry hypothesis in relation to emotion and behavior rests on the claim (perhaps first advanced by Davidson, 1992) that greater activation of the left frontal cortex is associated with greater approach motivation, which can include negative and positive affects. On the other hand, a predominance of the right frontal cortex indicates withdrawal motivation.

Both of these explanatory accounts of frontal asymmetry are relevant to depression. In the older account (Tomarken et al., 1992), frontal asymmetry is a marker of depression insofar as negative affects predominant over positive affects. In the newer account (Harmon-Jones, 2003; Harmon-Jones et al., 2010), frontal asymmetry's role in depression is to promote withdrawal over approach. Both of these accounts complement the American Psychological Association's (APA, 2013) description of the symptomatology of depression. According to the APA, depression combines negative affects (such as mood) with a lack of arousal (as related to thought and behavior) and withdrawal from hitherto pleasurable activities. However, even though the affect-based account of frontal asymmetry appears to be compatible with the symptomatology of depression, it is the approach- and withdrawalrelated account of frontal asymmetry that has come to be accepted in the context of applied psychology (Briesemeister et al., 2013). Briesemeister et al. (2013) conducted a meta-analysis of psychological experiments and found that frontal alpha asymmetry existed in $73.4 \%$ of scenarios in which subjects were confronted with an approach/withdrawal task, but only in $19.6 \%$ of scenarios in which no task was present.

If frontal alpha asymmetry is associated with increased withdrawal or negative affect, then increased frontal alpha symmetry should be associated with increased arousal or positive affect, which, in turn, should be associated with a reduction in depression symptoms. However, the metaanalysis conducted by Van Der Vinne et al. (2017) did not find a statistically significant effect of the existence of frontal alpha asymmetry on the diagnosis of depression, raising an important question about whether frontal alpha asymmetry has diagnostic validity with respect to MDD and other depression-related disorders. On the other hand, 
several of the studies cited by Briesemeister et al. (2013) suggested that frontal alpha asymmetry has predictive validity with respect to depression. In addition, diagnostic validity can be treated separately from the experimental question of whether, in the presence of treatment for depression, frontal alpha asymmetry is observed to decline.

Frontal alpha asymmetry, being measurable through electroencephalograms (EEG) collected in the context of NF research and interventions, can be reviewed in light of several of the studies included in the statistical meta-analysis whose results are presented above. Before such a discussion, it should be noted that there is a possible ambiguity in how the concept of asymmetry is approached. In some measurement approaches, an asymmetry increase is coded so as to reflect the reduced activation of the right frontal area; here, an asymmetry increase has the same functional meaning as an asymmetry decrease, that is, the state of the left and right frontal areas converging rather than diverging. Increasing asymmetry can mean reducing the activation of the right frontal area relative to the left, and decreasing asymmetry can mean achieving a state in which the left and right frontal areas converge in activation. Thus, even though the same underlying phenomenon is being measured, the manner in which it is conceptually and operationally approached can result in ambiguities in terminology. For the sake of clarity, the concept of asymmetry decrease has been applied to the various NF studies on this topic, with an asymmetry decrease representing the convergence of hyperactivated right frontal areas to the activation levels of left frontal areas.

Wang et al. (2019) reported that 4 out of 7 subjects who received NF designed to reduce frontal alpha asymmetry experienced a symmetry reduction, whereas the remaining participants experienced increased asymmetry. Wang et al. coded asymmetry reduction as an increase in the relative activation of alpha in the right frontal lobe. One of the special points in interest in Wang et al.'s study is that the individuals who experienced frontal alpha asymmetry reduction were also those who experienced a significant decline in depression; the individuals who did not experience frontal alpha asymmetry reduction also did not report a decline in depression. This finding was not reflected in Mennella et al.'s (2017) study, in which changes in frontal alpha asymmetry were not correlated with depression. However, unlike Wang et al., Mennella et al. did not analyze high responders to frontal alpha asymmetry reduction as a separate subclass, which would have disclosed whether individuals with marked reductions in frontal alpha asymmetry subsequent to NF also happen to report significantly lower depression scores.

Research on the various possible interrelationships between depression, frontal alpha asymmetry, and $\mathrm{NF}$ is in its relative infancy. However, a review of the $\mathrm{NF}$ literature suggests that front alpha symmetry reduction is, by means of arousal-withdrawal theory (Davidson, 1992), a plausible mechanism for reducing depressive symptoms. Another commonly utilized NF approach to depression reduction, rtfMRI-NF, also deserves close consideration because of the frequency with which it has been applied in NF practice.

In discussing the neurophysiological basis for rtfMRI, Young et al. (2017) cited the importance of the amygdala, which, as they noted, is overactivated relative to negative stimuli and less activated with respect to positive stimuli (Suslow et al., 2010; Victor et al., 2010), resulting in an overall hypoactive state in depression. Young et al. cited two prior rtfMRI-NFbased interventions in which depression reduction appears to have been achieved by means of stimulating increased activity in the amygdala. In their baseline analysis, Young et al. first confirmed that the amygdala was hemodynamically hypoactive among depressed patients, then confirmed that amygdala regulation increased significantly among participants in the rtfMRI-NF group. Because depression decreased significantly among those exposed to rtfMRI-NF, Young et al. drew the conclusion that the primary mechanism for depression reduction was increased activation of the amygdala.

The arousal-withdrawal model (Davidson, 1992) of depression can explain the results obtained by Young et al. (2017) -as well as the results obtained by Takamura et al. (2020) and Zotev et al. (2020), which were also obtained in the context of a hypothesis about amygdala hypoactivity as a correlate and substrate of depression. The hypoactive amygdala is, in essence, both a cause and an epiphenomenon of withdrawal and disengagement. If so, then rendering the amygdala more active through rtfMRI-NF has the potential to reduce depression, as found in the recent research (Takamura et al., 2020; Young et al., 2017; Zotev et al., 2020). The theoretical story appertaining to NF, depression, and amygdala is, in this way, just as explanatorily compelling as the narrative that links $\mathrm{NF}$, depression, and frontal asymmetry. 


\section{Conclusion}

Depression is perhaps the most widespread mental illness or disorder in the world (James et al., 2018) and is responsible for immense economic, social, and individual costs (Bubonya et al., 2017; Jia et al., 2015; Shin et al., 2020). Although there is consensus on the need to reduce depression, the question of how to achieve this reduction is increasingly open to challenge, redefinition, and exploration. Although there is no doubt that, on the whole, antidepressant pharmaceuticals work (Young \& Moulton, 2020), there is also compelling evidence that nonpharmaceutical interventions work (Kim, 2015). The contribution of this study was to demonstrate that NF is also an effective depressionreducing modality through the calculation of a pooled effect size, $g$, for numerous studies on depression and NF. In addition, the discussion of the underlying explanations for the efficacy of NF in this context called attention to the relevance of the arousal-withdrawal model of depression as it applies to frontal asymmetry and the activation of the amygdala. The effectiveness of NF against depression is not only observationally attestable through meta-analysis but theoretically describable through existing, well-supported models of depression and brain function. However, given the immaturity of the research base on NF, more work is necessary to deepen the claim for the effectiveness of this modality as either an alternative or a complement to pharmaceutical antidepressants.

\section{Author Declaration}

I have no conflicts, grants, or financial support to report relative to this article.

\section{References}

American Psychiatric Association. (2013). Diagnostic and statistical manual of mental disorders (5th ed.). https://doi.org /10.1176/appi.books.9780890425596

Baxter, A. J., Scott, K. M., Ferrari, A. J., Norman, R. E., Vos, T., \& Whiteford, H. A. (2014). Challenging the myth of an "epidemic" of common mental disorders: Trends in the global prevalence of anxiety and depression between 1990 and 2010. Depression and Anxiety, 31(6), 506-516. https://doi.org $/ 10.1002 /$ da. 22230

Beck, A. T., Steer, R. A., \& Brown, G. (1996). Beck Depression Inventory-II [Database record]. APA PsycTests. https://doi.org/10.1037/t00742-000

Briesemeister, B. B., Tamm, S., Heine, A., \& Jacobs, A. M. (2013). Approach the good, withdraw from the bad-a review on frontal alpha asymmetry measures in applied psychological research. Psychology, 4(3A), 261-267. https://doi.org/10.4236/psych.2013.43A039

Bubonya, M., Cobb-Clark, D. A., \& Wooden, M. (2017). Mental health and productivity at work: Does what you do matter? Labour Economics, 46, 150-165. https://doi.org/10.1016 /j.labeco.2017.05.001
Cheon, E.-J., Koo, B.-H., \& Choi, J.-H. (2016). The efficacy of neurofeedback in patients with major depressive disorder: An open labeled prospective study. Applied Psychophysiology and Biofeedback, 41(1), 103-110. https://doi.org/10.1007 /s10484-015-9315-8

Choi, S. W., Chi, S. E., Chung, S. Y., Kim, J. W., Ahn, C. Y., \& Kim, H. T. (2011). Is alpha wave neurofeedback effective with randomized clinical trials in depression? A pilot study. Neuropsychobiology, 63(1), 43-51. https://doi.org/10.1159 1000322290

Cohen, J. (2013). Statistical power analysis for the behavioral sciences. Cambridge, MA: Academic Press.

Davidson, R. J. (1992). Anterior cerebral asymmetry and the nature of emotion. Brain and Cognition, 20(1), 125-151. https://doi.org/10.1016/0278-2626(92)90065-T

Harmon-Jones, E. (2003). Clarifying the emotive functions of asymmetrical frontal cortical activity. Psychophysiology, 40(6), 838-848. https://doi.org/10.1111/1469-8986.00121

Harmon-Jones, E., Gable, P. A., \& Peterson, C. K. (2010). The role of asymmetric frontal cortical activity in emotion-related phenomena: A review and update. Biological Psychology, 84(3), $\quad 451-462 . \quad$ https://doi.org/10.1016 /j.biopsycho.2009.08.010

Harris, S., Hundley, G., \& Lambie, G. (2021). The effects of neurofeedback on depression, anxiety, and academic selfefficacy. Journal of College Student Psychotherapy, 35(1), 15-29. https://doi.org/10.1080/87568225.2019.1606689

Hou, Y., Zhang, S., Li, N., Huang, Z., Wang, L., \& Wang, Y. (2021). Neurofeedback training improves anxiety trait and depressive symptom in GAD. Brain and Behavior, 11(3), e02024. Advance online publication. https://doi.org/10.1002 /brb3.2024

James, S. L., Abate, D., Abate, K. H., Abay, S. M., Abbafati, C., Abbasi, N., Abbastabar, H., Abd-Allah, F., Abdela, J., Abdelalim, A., Abdollahpour, I., Abdulkader, R. S., Abebe, Z., Abera, S. F., Abil, O. Z., Abraha, H. N., Abu-Raddad, L. J., Abu-Rmeileh, N. M. E., Accrombessi, M. M. K., Acharya, D., ... Murray, C. J. L. (2018). Global, regional, and national incidence, prevalence, and years lived with disability for 354 diseases and injuries for 195 countries and territories, 19902017: A systematic analysis for the Global Burden of Disease Study 2017. The Lancet, 392(10159), 1789-1858. https://doi.org/10.1016/S0140-6736(18)32279-7

Jia, H., Zack, M. M., Thompson, W. W., Crosby, A. E., \& Gottesman, I. I. (2015). Impact of depression on qualityadjusted life expectancy (QALE) directly as well as indirectly through suicide. Social Psychiatry and Psychiatric Epidemiology, 50(6), 939-949. https://doi.org/10.1007 /s00127-015-1019-0

Kelly, B. D. (2020). Psychiatry's future: Biology, psychology, legislation, and "the fierce urgency of now." Indian Journal of Psychological Medicine, 42(2), 189-192. https://dx.doi.org $110.4103 \% 2 F I J P S Y M . I J P S Y M \_492 \_19$

Kim, J.-H., \& Kim, S.-Y. (2015). Effects of neurofeedback training on unilateral neglect and depression in patients with stroke. Journal of Digital Convergence, 13(9), 361-368. https://doi.org/10.14400/JDC.2015.13.9.361

Kim, Y. (2015). Preface. In Y. Kim (Ed.), Major depressive order: Cognitive and neurobiological mechanisms (pp. ix-x). Rijeka, Croatia: IntechOpen

Lenart, L., \& Fekete, A. (2021). Angiotensin receptor 1 blockade as an antidepression strategy. In C. Martin, L.-A. Hunter, V. Patel, V. Preedy, \& R. Rajendram (Eds.), The neuroscience of depression (pp. 283-292). London, UK: Academic Press.

Mennella, R., Patron, E., \& Palomba, D. (2017). Frontal alpha asymmetry neurofeedback for the reduction of negative affect and anxiety. Behaviour Research and Therapy, 92, 32-40. https://doi.org/10.1016/j.brat.2017.02.002

Peng, C.-Y. J., \& Chen, L.-T. (2014). Beyond Cohen's $d$ : Alternative effect size measures for between-subject designs. 
The Journal of Experimental Education, 82(1), 22-50. https://doi.org/10.1080/00220973.2012.745471

Sathappan, A. V., Luber, B. M., \& Lisanby, S. H. (2019). The dynamic duo: Combining noninvasive brain stimulation with cognitive interventions. Progress in NeuroPsychopharmacology and Biological Psychiatry, 89, 347-360. https://doi.org/10.1016/j.pnpbp.2018.10.006

Schwartz, J. M., Stapp, H. P., \& Beauregard, M. (2005). Quantum physics in neuroscience and psychology: A neurophysical model of mind-brain interaction. Philosophical Transactions of the Royal Society B: Biological Sciences, 360(1458), 1309-1327. https://doi.org/10.1098/rstb.2004.1598

Shin, D., Kim, N. W., Kim, M. J., Rhee, S. J., Park, C. H. K., Kim, H., Yang, B. R., Kim, M.-S., Choi, G. J., Koh, M, ... Ahn, Y. M. (2020). Cost analysis of depression using the national insurance system in South Korea: A comparison of depression and treatment-resistant depression. BMC Health Services Research, 20(286), 1-11. https://doi.org/10.1186 /s12913-020-05153-1

Suslow, T., Konrad, C., Kugel, H., Rumstadt, D., Zwitserlood, P., Schöning, S., Ohrmann, P., Bauer, J., Pyka, M., Kersting, A., Arolt, V., Heindel, W., \& Dannlowski, U. (2010). Automatic mood-congruent amygdala responses to masked facial expressions in major depression. Biological Psychiatry, 67(2), 155-160. https://doi.org/10.1016/j.biopsych.2009.07.023

Takamura, M., Okamoto, Y., Shibasaki, C., Yoshino, A., Okada, G., Ichikawa, N., \& Yamawaki, S. (2020). Antidepressive effect of left dorsolateral prefrontal cortex neurofeedback in patients with major depressive disorder: A preliminary report. Journal of Affective Disorders, 271, 224-227. https://doi.org /10.1016/j.jad.2020.03.080

Tomarken, A. J., Davidson, R. J., Wheeler, R. E., \& Doss, R. C. (1992). Individual differences in anterior brain asymmetry and fundamental dimensions of emotion. Journal of Personality and Social Psychology, 62(4), 676-687. https://doi.org /10.1037/0022-3514.62.4.676

Van Der Vinne, N., Vollebregt, M. A., Van Putten, M. J. A. M., \& Arns, M. (2017). Frontal alpha asymmetry as a diagnostic marker in depression: Fact or fiction? A meta-analysis. Neurolmage: Clinical, 16, 79-87. https://doi.org/10.1016 /j.nicl.2017.07.006
Victor, T. A., Furey, M. L., Fromm, S. J., Öhman, A., \& Drevets, W. C. (2010). Relationship between amygdala responses to masked faces and mood state and treatment in major depressive disorder. Archives of General Psychiatry, 67(11), 1128-1138. /archgenpsychiatry.2010.144 https://doi.org/10.1001

Wang, S.-Y., Lin, I.-M., Fan, S.-Y., Tsai, Y.-C., Yen, C.-F., Yeh, Y.-C., Huang, M.-F., Lee, Y., Chiu, N.-M., Hung, C.-F., Wang, P.-W., Liu, T.-L., \& Lin, H.-C. (2019). The effects of alpha asymmetry and high-beta down-training neurofeedback for patients with the major depressive disorder and anxiety symptoms. Journal of Affective Disorders, 257, 287-296. https://doi.org/10.1016/j.jad.2019.07.026

Young, A. H., \& Moulton, C. D. (2020). Antidepressants do work after all. Journal of Psychopharmacology, 34(10), 1071-1073. https://doi.org/10.1177/0269881120933127

Young, K. D., Siegle, G. J., Zotev, V., Phillips, R., Misaki, M., Yuan, H., Drevets, W. C., \& Bodurka, J. (2017). Randomized clinical trial of real-time fMRI amygdala neurofeedback for major depressive disorder: Effects on symptoms and autobiographical memory recall. The American Journal of Psychiatry, 174(8), 748-755. https://doi.org/10.1176 /appi.ajp.2017.16060637

Yu, S. H., Tseng, C. Y., \& Lin, W. L. (2020). A neurofeedback protocol for executive function to reduce depression and rumination: A controlled study. Clinical Psychopharmacology and Neuroscience: The Official Scientific Journal of the Korean College of Neuropsychopharmacology, 18(3), 375385. https://doi.org /10.9758/cpn.2020.18.3.375

Zotev, V., Mayeli, A., Misaki, M., \& Bodurka, J. (2020). Emotion self-regulation training in major depressive disorder using simultaneous real-time fMRI and EEG neurofeedback. Neurolmage: Clinical, 27, 102331. https://doi.org/10.1016 /j.nicl.2020.102331

Received: March 28, 2021

Accepted: April 21, 2021

Published: June 30, 2021 